\title{
Simulative Verification of Channel Reciprocity in Free-Space Optical Inter-HAP Links
}

\author{
Swaminathan Parthasarathy, Dirk Giggenbach, \\ Ricardo Barrios, Christian Fuchs \\ Institute of Communications and Navigation (IKN) \\ German Aerospace Center (DLR) \\ Oberpfaffenhofen, 82234 Wessling, Germany
}

\author{
Andreas Kirstädter \\ Institute of Communication Networks and Computer \\ Engineering (IKR) \\ University of Stuttgart \\ Pfaffenwaldring 47, 70569 Stuttgart, Germany
}

\begin{abstract}
Channel reciprocity in a bidirectional turbulent channel implies that the received powers are correlated to a certain extent at both link ends. In this paper, we investigate by numerical phase-screen propagation simulation the variation of the channel correlation under various boundary conditions, namely orthogonal wind speed, link distance, receiver aperture and turbulence-strength profile for inter High Altitude Platform (HAP) FSO links. Finally, we provide an outlook to different Error Control Coding (ECC) methods that make use of the reciprocity effect.
\end{abstract}

Keywords-Stratosphere; Atmospheric turbulence; correlation; Error Control Coding; CSI; PIB

\section{INTRODUCTION}

Directed optical free-space links are the privileged technique for high-speed data interconnects in future stratospheric communication platform networks, with so-called High Altitude Platforms (HAPs) [1]-[4]. Applied in stratospheric altitudes, this data transmission method enables transmission rates of several tens of gigabits per second via single or multiple WDM channels over several hundred kilometer link distance. While the atmospheric transmission medium is highly linear and non-dispersive - thereby avoiding the degrading effects of optical fibers - the remaining index-ofrefraction turbulence at stratospheric altitudes causes medium to strong scintillation of the optical intensity after transmission over such long horizontal links, generating fades and surges of received power [5].

The temporal behavior of these scintillations is governed by the turbulence cell structure sizes and the wind speed orthogonal to the link direction. As in similar experiments and simulations, one has to expect channel coherence time (given by scintillation speed) in the order of few milliseconds, defined by the autocovariance half-width half-maximum of received power $\left(\tau_{\text {acv }}\right)$. If perfect Channel State Information (CSI) of the received power could be exchanged without delay between sender and receiver, the available channel capacity could be exploited by according error control techniques. However, $\tau_{a c v}$ happens to be also in the order of the time-of-flight $(\mathrm{ToF})-t_{T O F}$ - of the optical signal between both nodes in a single link, when the two HAPs are typically positioned some $100 \mathrm{~km}$ apart. Thus, any exchange of received CSI to control scintillation losses is prone to being outdated due to processing time plus $t_{T o F}$, depending on link scenario specifics, see Fig. 1. In a symmetric monostatic link geometry - i.e. when transmit and received beam of each terminal share the same optical axis and aperture sizes - on-axis intensity-reciprocity can be observed [6]. This axial intensity reciprocity is extended to spatial diameters below the intensity speckle patches, allowing received power reciprocity with reasonably sized receiver apertures [7]-[11]. The quality of this power reciprocity is defined by the correlation coefficient (CCF) of the received power vectors at both link ends [8][9]. Specifically, in typical inter-HAP links, these speckle patches tend to be in the order of several decimeters, allowing equivalent receiver apertures, large enough to support high-speed links while still offering high CCF close to one.

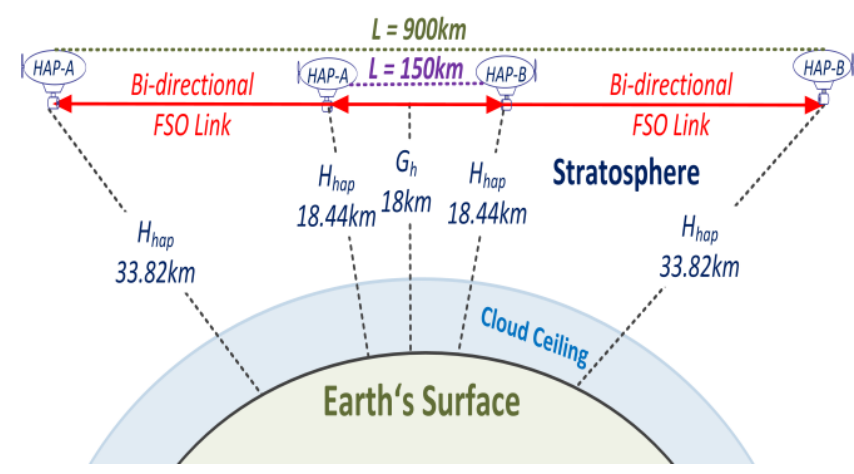

Fig. 1. Bi-directional inter-HAP FSO link scenario at the stratosphere between HAPs Aand B (distances not to scale).

This inherent CSI by reciprocity then can be employed for optimizing Error Control Coding (ECC) on the link accordingly. In reality, however also in case of ideal static atmospheric conditions - allowing a CCF close to one - the signal's ToF between both nodes leads to an aging of the CSI. Furthermore, the changes in turbulence structure - caused by orthogonal winds moving turbulence cells through the beams can alter the channel during $t_{T O F}$ such that both directions do not experience the same absolute turbulence effects at the same location, leading to a reduced CCF. 
In this paper, verification of reciprocity between the received powers at both ends of bi-directional free-space optical inter-HAP links is presented. We investigate by numerical phase-screen propagation simulation the variation of the channel reciprocity under various boundary conditions, namely orthogonal wind speed, link distance, and receiver aperture. We investigate the so called power-in-the-bucket (PIB) reciprocity that uses multi-mode receivers which measure the entire optical signal power entering the focusing lens apertures. Finally, we provide a brief outlook to different Error Control Coding (ECC) methods that make use of the reciprocity effect.

The remainder of this paper is organized as follows: The simulation concept of numerical phase-screen propagation is described in Section II. In Section III, the chosen scenarios and channel model are presented in detail. Simulation results are presented and discussed in detail in Section IV. Finally, Section $\mathrm{V}$ concludes this paper.

\section{PILAB Simulation CONCEPT}

PILab (Propagation and Imaging Lab) is a Matlab based programming tool to simulate FSO communication scenarios [12][13]. It uses atmospheric propagation simulations in which the spatial and temporal dynamics of the atmospheric turbulence are modelled by phase screens that are shifted laterally according to the orthogonal wind. The temporal fluctuations of the received power signals are induced due to orthogonal winds. This section focuses only on the simulation of reciprocal inter-HAP FSO channels. To implement the wind shifting during the propagation of the beam, each phase screen is moved by a number of pixels

$$
N_{x}=\frac{d * v_{x}}{c * \delta_{x}}, N_{y}=\frac{d * v_{y}}{c * \delta_{y}}
$$

Here, $N_{x}$ and $N_{y}$ are the number of pixels to shift along the directions $x$ and $y$ respectively. $v_{x}$ and $v_{y}$ are the wind speeds projected to the directions $x$ and $y$. The speed of light in the earth atmosphere (considered equal to the speed of light in the vacuum) is given by $c, d$ is the distance of a phase screen from the transmitter. $\delta_{x}$ and $\delta_{y}$ are the resolutions along $x$ and $y$ of the image.

The simulation of the reciprocal channels was done in two steps:

- The first step is to create a reciprocal channel by using the same phase screens for both the forward and return path while taking care of reverting the order in the latter. In other words, the first phase screen for the forward direction becomes the last phase screen for the return path, the second phase screen for the forward path become the second last for the backward direction and so on for all the phase screens.

- Then, the phase screens of the return path are flipped to emulate the respective point of view of the scene for each transmitter. Moreover, during the simulation time the shifting of the phase screens due to the wind, for the backward direction is the opposite of that of the forward path.

As the aim is to replicate the exact same phase screens for the return channel, the extent of the propagating 2D array is kept constant. Nevertheless, as the beam propagates its physical extent may exceed that of the generated turbulent phase screens. When the beam becomes larger than the phase screen extent, energy is reflected from the edges producing self-interference patterns that translate into image aliasing problems. To avoid this, a damper is placed before the diffracted field extent approaches that of the phase screens. Finally, the field source at the transmitter plane is a Gaussian beam of the corresponding size, immediately followed by a lens element in the simulator to produce a non-collimated beam that approaches the diffraction behavior of a spherical wave. The method used is based on the proposal of Martin and Flatte for the simulation of point-sources presented in [14].

\section{SCENARIO DEFINITION}

\section{A. Inter-HAP}

The investigated bi-directional inter-HAP scenarios are presented in TABLE II. We considered eight different link distances $(L)$ for HAPs positioned at different heights so called as HAP heights $\left(H_{h a p}\right)$ and assuming one graze height $\left(G_{h}\right)$ for all scenarios. $G_{h}$ refers to the minimum height of the optical link above the earth surface of the earth determined by the cloud ceiling [2], where the turbulence is maximum. The propagation delay for different link distances/ time-of-flight (ToF) is given by $t_{T o F}$. We have investigated scenarios with different HAP heights and link distances to show the consequences of different atmospheric and geometric parameters. An example of two bidirectional inter-HAP link scenarios is depicted in Fig. 1.

TABLE I. INTER-HAP SCENARIOS

\begin{tabular}{|c|c|c|c|}
\hline $\begin{array}{c}\text { Link } \\
\text { Distance } \\
\mathbf{L} \\
(\mathbf{k m}) \\
\end{array}$ & $\begin{array}{c}\mathbf{t}_{\mathrm{ToF}} \\
(\mathrm{ms})\end{array}$ & $\begin{array}{c}\text { Graze } \\
\text { Height }^{\text {a }} \\
\mathbf{G}_{\mathbf{h}} \\
(\mathbf{k m}) \\
\end{array}$ & $\begin{array}{c}\text { HAP } \\
\text { Height } \\
\text { H }_{\text {hap }} \\
(\mathbf{k m})\end{array}$ \\
\hline 150 & 0.5 & \multirow{8}{*}{18} & 18.44 \\
\hline 200 & 0.66 & & 18.78 \\
\hline 300 & 1 & & 19.76 \\
\hline 450 & 1.33 & & 21.96 \\
\hline 600 & 2 & & 25.09 \\
\hline 700 & 2.33 & & 25.57 \\
\hline 800 & 2.66 & & 30.51 \\
\hline 900 & 3 & & 33.82 \\
\hline
\end{tabular}

a. minimum height of the optical link above the surface of the earth (maximum turbulence regime) 


\section{B. Optical Turbulence and Path Profile}

Atmospheric turbulence is often characterized by the parameter $C_{n}^{2}$ (in units of $\mathrm{m}^{-2 / 3}$ ), called the refractive index structure parameter. The altitude profile for the $C_{n}{ }^{2}$ parameter is calculated using the Hufnagel-Valley $(\mathrm{H}-\mathrm{V})$ model [5] given by:

$$
\begin{gathered}
C_{n}^{2}(h)=0.00594\left(\frac{w}{27}\right)^{2}\left(10^{-5} h\right)^{10} \exp \left(-\frac{h}{1000}\right)+2.7 \times \\
10^{-16} \exp \left(-\frac{h}{1500}\right)+A \exp \left(-\frac{h}{100}\right)
\end{gathered}
$$

where $h$ is the altitude of the link in meters, $w$ is the RMS wind speed (pseudo wind) in $\mathrm{m} / \mathrm{s}$ and $A$ is the value of $C_{n}^{2}$ at $h=0$ in $m^{-2 / 3}$. In our calculations, we use the $\mathrm{H}-\mathrm{V}$ model with $w$ $=21 \mathrm{~m} / \mathrm{s}$ and $A=1.7 \times 10^{-14} \mathrm{~m}^{-2 / 3}$, commonly known as the H$\mathrm{V}_{5 / 7}$ model [5]. The RMS wind speed shall not be confused with link orthogonal wind speed $(v)$.

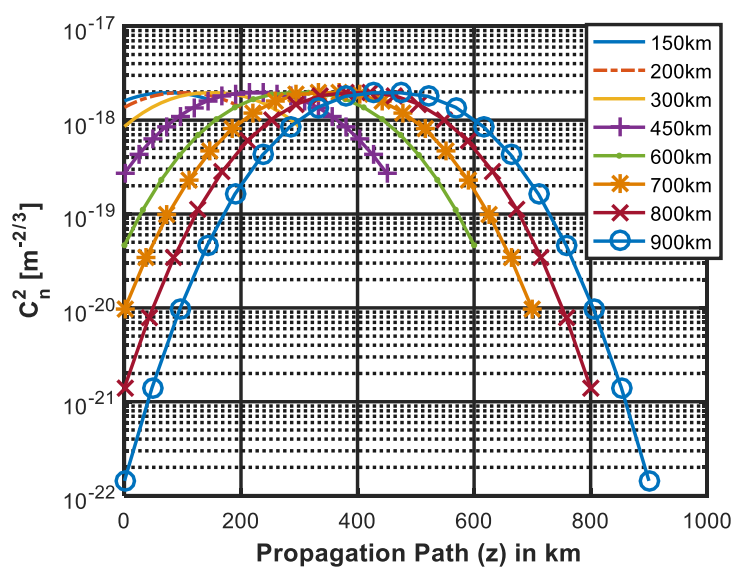

Fig. 2. Turbulence strength $\left(\mathrm{C}_{\mathrm{n}}{ }^{2}\right)$ along the propagation path for different link distances (L)

Fig. 2 shows the calculated $\mathrm{C}_{\mathrm{n}}{ }^{2}$-profiles using equation (3) for different inter-HAP scenarios along the propagation path of FSO link. The maximum value of $\mathrm{C}_{\mathrm{n}}{ }^{2}$ along the Inter-HAP link path is seen in the middle of the link and the average value of $\mathrm{C}_{\mathrm{n}}{ }^{2}$-profile increases with the link distance.

\section{Numerical Parameters}

The performance of an Inter-HAP FSO communication system can be significantly reduced by turbulence-induced scintillation resulting from beam propagation through the atmosphere. Specifically, scintillation can lead to power losses at the receiver and eventually to fading of the received signal below a detectable threshold. The laser beam propagating through the atmosphere will experience intensity fluctuations due to turbulence induced Index of Refraction (IRT) known as scintillations.

The spherical wave Rytov variance is $\beta_{0}{ }^{2}=0.4{\sigma_{R}}^{2}$, where $\sigma_{R}^{2}$ is the plane wave Rytov variance and the normalized variance of received power Pinto a given receiver aperture diameter $\left(D_{r x}\right)$ is the Power Scintillation Index (PSI) given by [5]:

$$
\sigma_{I}^{2}\left(D_{r x}\right)=\frac{\left\langle P^{2}\right\rangle-\langle P\rangle^{2}}{\langle P\rangle^{2}}
$$

where $\langle$.$\rangle represents time averaging. PSI in our scenario$ represents the strength of atmospheric turbulence with respect to the simulated normalized received power $(P)$ for a given aperture $\left(D_{r x}\right)$. The simulated vectors at $\mathrm{A}$ and $\mathrm{B}$ are compared in terms of Correlation Coefficient (CCF) and Normalized Mean Squared Error (NMSE) are defined as follows [15][16]:

$$
\begin{aligned}
& C C F=\frac{E\left\{\left(A_{i}-\mu_{A}\right)\left(B_{i}-\mu_{B}\right)\right\}}{\sigma_{A} \sigma_{B}} \\
& N M S E=\frac{\sum_{i=1}^{N}\left(A_{i}-B_{i}\right)^{2}}{\mu_{A} \mu_{B}}
\end{aligned}
$$

where $A_{i}$ and $B_{i}$ are received optical powers over time measured at terminals $A$ and $B$, and $\mu$ and $\sigma$ represent their means and standard deviations respectively. Both CCF and NMSE together are used here as metric to evaluate the quality of channel reciprocity. The reason is that NMSE regards only absolute power variations. CCFs below 1 or NMSE above 0 respectively represent the real-world imperfectness of the reciprocity of channel state information.

\section{RESULTS AND DISCUSSION}

In this section, we present the simulation results illustrating the performance of reciprocal inter-HAP FSO links for our scenarios shown in TABLE I. For all scenarios, we performed an averaging of 5 (extremely time-consuming simulation runs) time series, resulting in a total power vector length of $10 \mathrm{~s}$. We evaluate numerical simulation results based on the following parameters such as Power Scintillation Index (PSI), Correlation Coefficient (CCF) and Normalized Mean Squared Error (NMSE). TABLE II. shows a summary of simulation results for different link distances for two different input orthogonal wind speeds (v) $10 \mathrm{~m} / \mathrm{s}$ and $50 \mathrm{~m} / \mathrm{s}$. The output parameters of the simulation are for one value of receiver aperture diameter $\left(D_{r x}\right) 20 \mathrm{~cm}$.

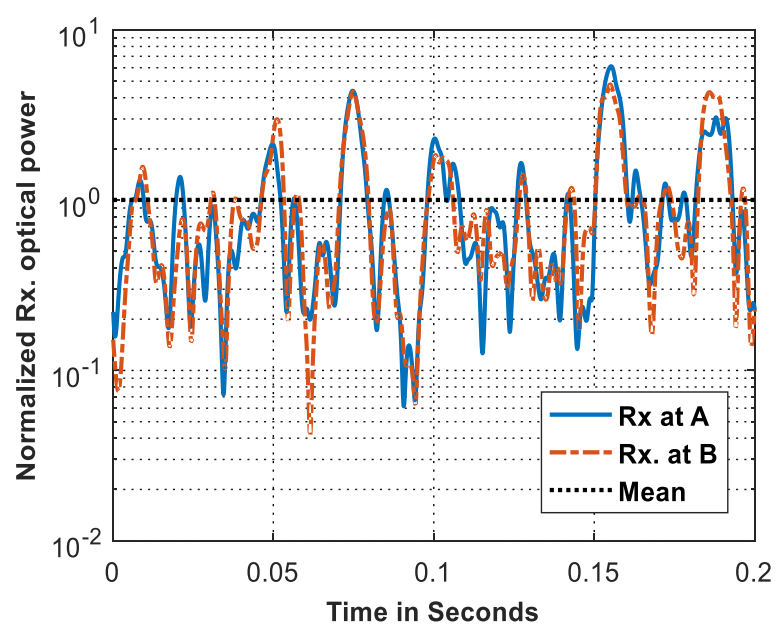

Fig. 3. Timeseries of first $200 \mathrm{~ms}$ of simulated normalized received power vectors for $900 \mathrm{~km}$ for $\mathrm{D}_{\mathrm{rx}}=20 \mathrm{~cm} . \mathrm{CCF}=0.924, \mathrm{NMSE}=0.18$, PSI-A 
$=0.981$, PSI-B $=1.126$, HWHM acov-A $=2.95 \mathrm{~ms}$, HWHM acov-B $=$

$2.91 \mathrm{~ms}$.

TABLE II. SUMMARY OF INTER-HAP SIMULATION RESULTS

\begin{tabular}{|c|c|c|c|c|c|c|c|c|}
\hline \multirow{2}{*}{$\begin{array}{c}\text { Link } \\
\text { Distance } \\
\text { L } \\
(\mathbf{k m})\end{array}$} & \multirow{2}{*}{$\begin{array}{c}\mathbf{t}_{\mathrm{ToF}} \\
(\mathrm{ms})\end{array}$} & \multirow{2}{*}{$\begin{array}{l}\text { Wind } \\
\text { speed }^{\text {a }} \\
\mathbf{v} \\
(\mathbf{m} / \mathbf{s})\end{array}$} & \multicolumn{6}{|c|}{$\begin{array}{c}\text { Simulation Output Parameters } \\
\text { for } \\
D_{\mathrm{rx}}=20 \mathrm{~cm} \\
\end{array}$} \\
\hline & & & $\begin{array}{c}\text { PSI- } \\
A\end{array}$ & $\begin{array}{c}\text { PSI- } \\
B\end{array}$ & $\begin{array}{c}\text { HWHM } \\
\text { acov. - } \\
\text { A } \\
(m s)\end{array}$ & $\begin{array}{c}\text { HWHM } \\
\text { acov. - } \\
\text { B } \\
(\mathrm{ms})\end{array}$ & NMSE & $C C F$ \\
\hline \multirow{2}{*}{150} & \multirow{2}{*}{0.5} & 10 & 0.103 & 0.099 & 9.33 & 9.27 & 0.013 & 0.931 \\
\hline & & 50 & 0.109 & 0.113 & 17.3 & 17.7 & 0.013 & 0.937 \\
\hline \multirow{2}{*}{200} & \multirow{2}{*}{0.66} & 10 & 0.110 & \begin{tabular}{c|}
0.108 \\
\end{tabular} & 8.05 & 8.55 & 0.022 & 0.895 \\
\hline & & 50 & 0.217 & 0.189 & 2.45 & 2.35 & 0.019 & 0.953 \\
\hline \multirow{2}{*}{300} & \multirow{2}{*}{1} & 10 & 0.382 & 0.376 & 11.97 & 11.47 & 0.024 & 0.967 \\
\hline & & 50 & 0.369 & 0.369 & 2.13 & 2.13 & 0.037 & 0.948 \\
\hline \multirow{2}{*}{450} & \multirow{2}{*}{1.33} & 10 & 0.613 & 0.597 & 13.09 & 13.33 & 0.027 & 0.976 \\
\hline & & 50 & 0.547 & 0.529 & 2.39 & 2.41 & 0.065 & 0.932 \\
\hline \multirow{2}{*}{600} & \multirow{2}{*}{2} & 10 & 0.820 & 0.852 & 15.55 & 15.43 & 0.028 & 0.984 \\
\hline & & 50 & 0.807 & 0.773 & 2.89 & 2.83 & 0.077 & 0.949 \\
\hline \multirow{2}{*}{700} & \multirow{2}{*}{2.33} & 10 & 0.894 & 0.974 & 14.11 & 14.05 & 0.091 & 0.947 \\
\hline & & 50 & 0.991 & 1.084 & 2.77 & 2.73 & 0.173 & 0.926 \\
\hline \multirow{2}{*}{800} & \multirow{2}{*}{2.66} & 10 & 0.856 & 0.897 & 15.52 & 15.11 & 0.064 & 0.962 \\
\hline & & 50 & 0.898 & 0.924 & 3.29 & 2.67 & 0.128 & 0.942 \\
\hline \multirow{2}{*}{900} & \multirow{2}{*}{3} & 10 & 0.931 & 0.917 & 14.83 & 14.45 & 0.077 & 0.956 \\
\hline & & 50 & 0.981 & 1.126 & 2.95 & 2.91 & 0.181 & 0.924 \\
\hline
\end{tabular}

Fig. 3 depicts typical received optical powers at both terminals A and B, respectively. The powers received at A and B represent optical powers at HAPs A and B for a receiver aperture diameter $D_{r x}=20 \mathrm{~cm}$.

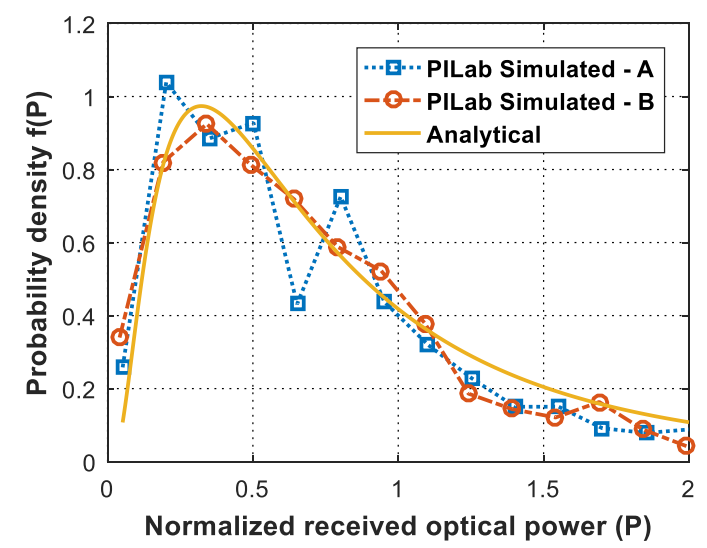

Fig. 4. Comparison of the received power PDFs as obtained from one PILab simulation run, and analytical (lognormal) distribution.

Fig. 4 shows an example of the comparison of PDF estimate calculated using lognormal distribution compared with the simulation results. We see that the analytical and simulated results match well for PSI $\sim 1.5$. The outliers of the PDF of the simulated values are due to the limited continuous vector length as given by the finite size of phase screens moved with the orthogonal wind speed. An overall summary of different scintillation indices for different link distances and an orthogonal wind speed of $50 \mathrm{~m} / \mathrm{s}$ is depicted in Fig. 5. ISI (Intensity Scintillation Index) refers to a point receiver. $\beta_{0}{ }^{2}$ calculated analytically shows the increase of Rytov variance along the link distance and the simulated ISIs and PSIs (for $D_{r x}$ $=20 \mathrm{~cm}$ ). In general, the scintillation index increases with the increase in the link distance $(L)$ as the turbulence strength increases with $L$ as shown in Fig. 2. The difference in the scintillation index values between ISI and PSI are also observed.

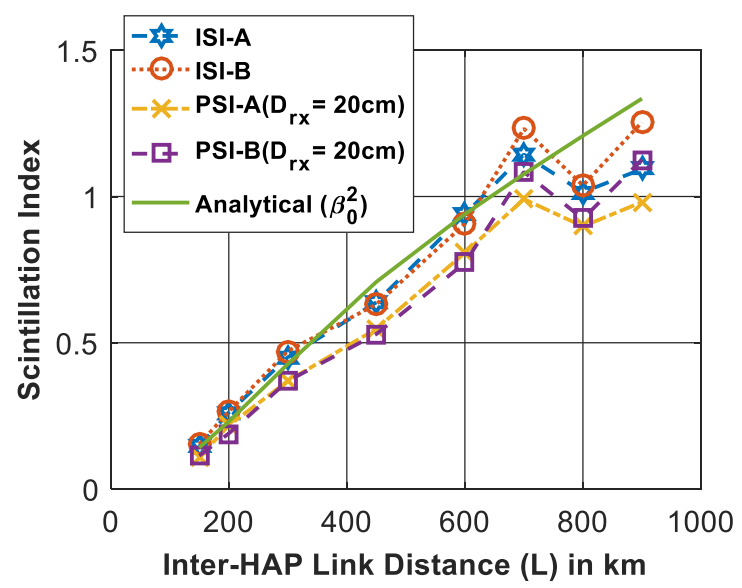

Fig. 5. Overall summary of scintillation indices for different inter-HAP link distances for orthogonal wind speed (v) of $50 \mathrm{~m} / \mathrm{s}$.

The intensity speckle width is directly related to the size of the first Fresnel zone given by $\rho_{c} \sim \sqrt{\lambda . L}$ for a collimated beam. $\rho_{\mathrm{c}}$ identifies the maximum receiver aperture size that will act like a point receiver. Aperture sizes larger than $\rho_{c}$ will experience aperture averaging, that in the end reduces scintillation [5]. Fig. 6 shows the increase in speckle size analytically $\left(\rho_{\mathrm{c}}\right)$ and simulated $\left(\rho_{\mathrm{i}}\right)$, with the link distance. Fig. 
7 shows CCF and NMSE for different inter-HAP link distances. The values are for two extreme orthogonal wind speeds $10 \mathrm{~m} / \mathrm{s}$ and $50 \mathrm{~m} / \mathrm{s}$. To quantify the quality of reciprocity one has to consider both CCF and NMSE values. We see that although there are minor variations of $\mathrm{CCF}$ along $L, \mathrm{NMSE}$ increases along $L$ representing reduced reciprocity effect with the increase in link distance $(L)$. We would expect an even stronger decrease of the reciprocity effect with larger distances due to the change of IRT-structure during long ToFs.

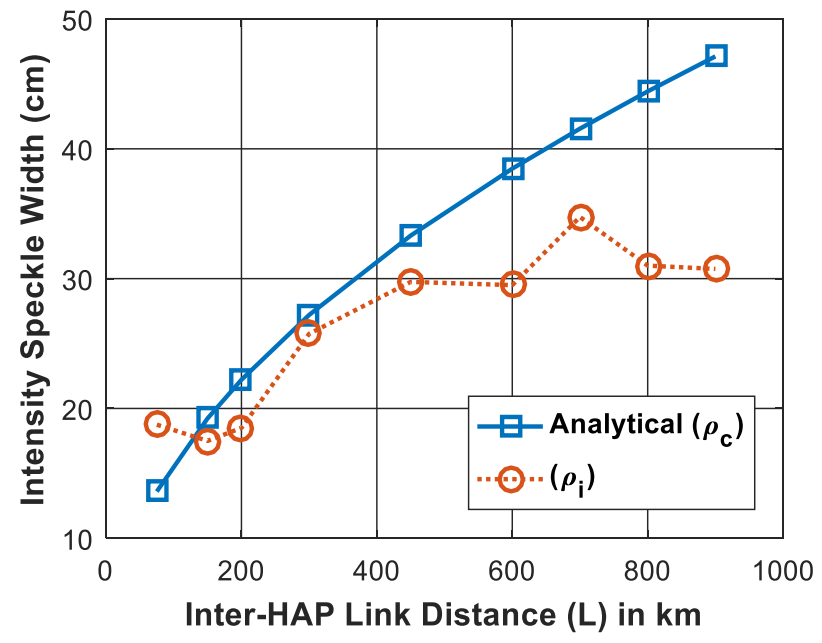

Fig. 6. Comparison of intensity speckle width (simulated and analytical) for different inter-HAP link distances.

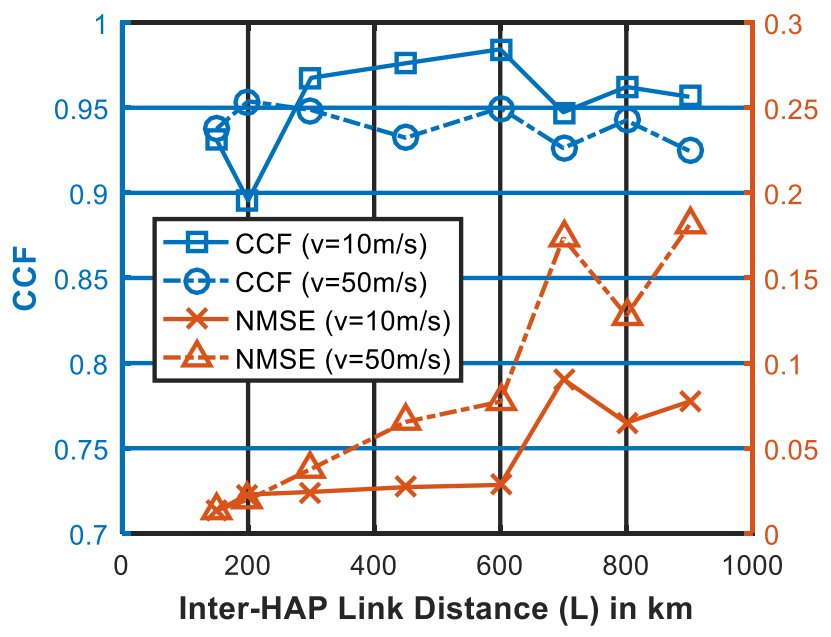

Fig. 7. CCF and NMSE for different orthogonal wind speeds (v) along different inter-HAP link distances.

In Fig. 8, the variations of CCF and NMSE for different link distances $(L)$ are shown as a function of $D_{r x} / \rho_{i}$. The reduction in CCF is observed as the $D_{r x} / \rho_{i}$ parameter increases and NMSE increases showing a reduction in overall reciprocal quality. We observe that for $D_{r x} / \rho_{i}=0$, the CCF is lower since the smaller receiver aperture is sensitive to orthogonal wind speed.

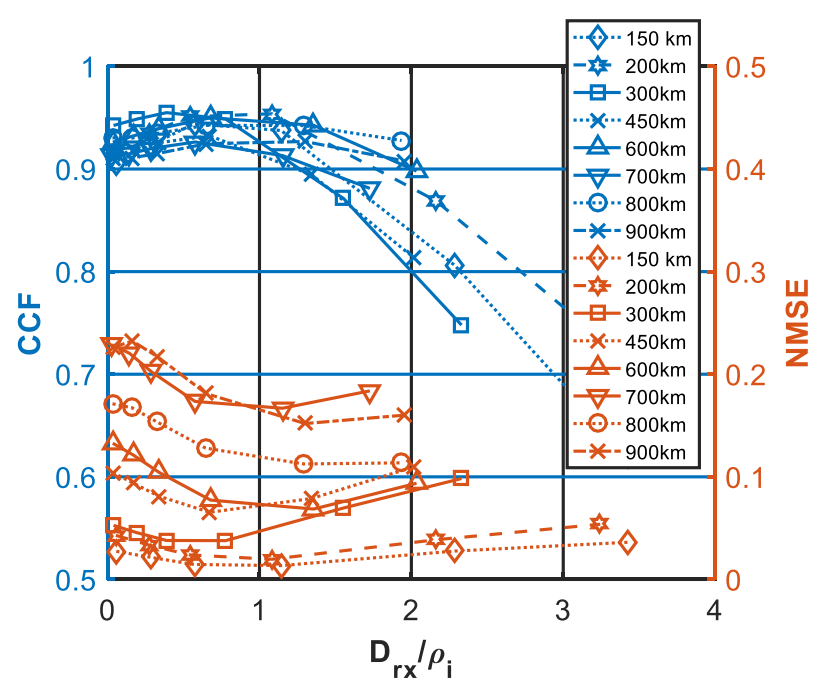

Fig. 8. CCF and NMSE as a function of normalized receiver aperture diameter $\left(D_{\mathrm{rx}}\right)$ for different inter-HAP link distances.

\section{CONCLUSION}

In this work, we investigated by numerical phase-screen propagation simulation the variations of FSO inter-HAP channel reciprocity under various boundary conditions, namely orthogonal wind speed, link distance, and receiver aperture size. The quality of reciprocity was quantified in terms of Correlation Coefficient (CCF) and Normalized Mean Squared Error (NMSE). A detailed summary of different output parameters was presented as simulation results. Our numerical simulations confirm the existence of strong reciprocity over reasonable receiver aperture diameters as applicable to inter-HAP links.

The reciprocity demonstrated also offers very promising opportunities for error control to overcome the effects of signal scintillation due to turbulence in atmospheric FSO links. Lowest-delay channel state information (CSI) is available as the transmitter inherently (so called "Reciprocal CSI") has the knowledge of the channel quality as currently experienced by the receiver. This CSI can be used in inter-HAP FSO links for adaptive link error control - adapting code-rate or data-rate within hybrid ARQ techniques [17][18].

Thus, the real-time property of reciprocal CSI partly compensates the negative effects of the long link delays in interHAP scenarios. This advantage especially extends to multi-hop topologies where excessive retransmissions on long-delay links would have a detrimental effect on the end-to-end delay performance.

\section{References}

[1] D. Grace and M. Mohorcic., Broadband Communications via HighAltitude Platforms, Springer, 2010.

[2] D. Giggenbach, R. Purvinskis, M. Werner, M. Holzbock, "Stratospheric Optical Inter-Platform Links for High Altitude Platforms," Online Proceedings. 20th AIAA International Communications Satellite Systems Conference, 2002.

[3] C. Chen, A. Grier, M. Malfa, E. Booen, H. Harding, C. Xia, M. Hunwardsen, J. Demers, K. Kudinov, G. Mak, B. Smith, A.Sahasrabudhe, F. Patawaran, T. Wang, A. Wang, C. Zhao, D. Leang, J. Gin, M. Lewis, D. Nguyen, K. Quirk, "High-speed optical links for UAV applications," 
Proc.SPIE 10096, Free-Space Laser Communication and Atmospheric Propagation XXIX, 1009615, March 7, 2017.

[4] B. Moision, B. Erkmen, E. Keyes, T. Belt, O. Bowen, D. Brinkley, P. Csonka, M. Eglington, A. Kazmierski, N. Kim, J. Moody, T. Tu, W. Vermeer, "Demonstration of free-space optical communication for longange data links between balloons on Project Loon," Proc. SPIE 10096 , Free-Space Laser Communication and Atmospheric Propagation XXIX, 100960Z, February 24, 2017.

[5] L.C. Andrews and R.C. Phillips., "Laser Beam Propagation through Random Media - Second Edition," SPIE-Press, Bellingham, 2005.

[6] J. H. Shapiro, "Reciprocity of the Turbulent Atmosphere*," J. Opt. Soc. Am., vol. 61, 1971, pp. 492-495.

[7] R. R. Parenti, J. M. Roth, J. H. Shapiro, F. G. Walther, and J. A. Greco, "Experimental observations of channel reciprocity in single-mode freespace optical links," Opt. Express 20, pp. 21635-21644, 2012.

[8] D. Giggenbach, W. Cowley, K. Grant, and N. Perlot, "Experimental verification of the limits of optical channel intensity reciprocity," Appl. Opt., vol. 51, pp. 3145-3152, 2012.

[9] N. Perlot and D. Giggenbach, "Scintillation correlation between forward and return spherical waves," Appl. Opt. 51, pp. 2888-2893, 2012.

[10] J. H. Shapiro and A. L. Puryear, "Reciprocity-enhanced optical communication through atmospheric turbulence-Part I: Reciprocity proofs and far-field power transfer optimization," in IEEE/OSA Journal of Optical Communications and Networking, vol. 4, no. 12, pp. 947-954, Dec. 2012.

[11] A. L. Puryear, J. H. Shapiro and R. R. Parenti, "Reciprocity-enhanced optical communication through atmospheric turbulence - Part II: Communication architectures and performance," in IEEE/OSA Journal of
Optical Communications and Networking, vol. 5, no. 8, pp. 888-900, Aug. 2013.

[12] J. Horwath, N. Perlot, D. Giggenbach, R. Jüngling, "Numerical simulations of beam propagation through optical turbulence for highaltitude platform crosslinks," Proc. SPIE, vol. 5338, Free-Space Laser Communication Technologies XVI, June.16, 2004, pp. 243.

[13] N. Perlot, J. Horwath, R. Jüngling, "Modeling wind in simulations of atmospheric optical propagation," Proc. SPIE, vol. 5712, Free-Space Laser Communication Technologies XVII, May 02, 2005, pp. 140.

[14] J. M. Martin and Stanley M. Flatté, "Simulation of point-source scintillation through three-dimensional random media," J. Opt. Soc. Am. A 7, 1990, 838-847

[15] Z. Wang and A. C. Bovik, "Mean squared error: Love it or leave it? A new look at Signal Fidelity Measures," IEEE Signal Processing Magazine, vol. 26, no. 1, Jan. 2009, pp. 98-117.

[16] L. Mandel and E. Wolf (1995). Optical Coherence and Quantum Optics. [Online]:https://www.cambridge.org/core/books/optical-coherence-andquantum-optics/F8CB94C70FA64CD3FB60890CA2048168.

[17] S. Parthasarathy, A. Kirstaedter and D. Giggenbach, "Performance Analysis of Adaptive Hybrid ARQ for Inter-HAP Free-Space Optical Fading Channel with Delayed Channel State Information," Photonic Networks; 17. ITG-Symposium; Proceedings of, Leipzig, Germany, 2016, pp. 1-7.

[18] S. Parthasarathy, A. Kirstaedter and D. Giggenbach, "Adaptive HARQ with Channel State Information in Inter-HAP FSO Links," Photonic Networks; 18. ITG-Symposium, Leipzig, Germany, 2017, pp. 1-6. 